\title{
A Numerical Analysis of Optimum Air Journal Bearings \\ Lie $\mathrm{KN}^{1 *}$ and Jerry C T Su${ }^{2}$
}

${ }^{1}$ Far East University, Tainan, 744, Taiwan ROC

${ }^{2}$ Department of Mechanical and Automation Engineering, National Kaohsiung First University of Science and Technology, Kaohsiung, 824, Taiwan ROC

\begin{abstract}
The responses to changes of bearing length-to-diameter ratio and supply pressure of hybrid air journal bearings are investigated numerically for rotor dynamic instability. Different types of external pressure compensations, including multi-array of 1, 2, 3, 4, or 5-row orifice bearings and porous bearings, are analyzed to obtain more insight to optimum designs of journal bearings to improve the problem of whirl instability of rotor mass. The results show that the long porous bearings $(L / D>1.0)$ in the higher rotation speeds $(\Lambda>0.5)$ have higher threshold load capacities $W$ before the onset of whirl instability and thus are more stable than orifice bearings. On the contrary, the short 5-row orifice bearings $(L / D \leq 1.0)$ are more stable than the porous bearing in the lower rotation speeds $(0.1 \leq \Lambda \leq 0.5)$. The results also show that the change of supply pressure from $P=2.0$ to $P=8.0$ makes no difference to orifice bearing with whirl instability of rotor but that the porous bearing is the most stable for the lower supply pressure $P_{s}=2.0$ and becomes unstable as the supply pressure $P_{s}$ increases in the higher rotation speeds $(\wedge>0.5)$.
\end{abstract}

Keywords: Air journal bearing; Porous bearing; Rotor dynamic instability

\section{Nomenclature}

$a$ : radius of orifice

$B_{i j}$ dimensionless damping coefficient $=\frac{\bar{B}_{i j} C v}{2 \pi R L P_{a}}$

$\bar{B}_{i j}$ : damping coefficient

$C$ : concentric bearing clearance for $\varepsilon=0$

$C_{d}$ : orifice discharge coefficient

$C_{p}$ : specific heat of constant pressure of air

$C_{v}$ : specific heat of constant volume of air

$D$ : diameter of journal

$e$ : eccentricity

$F$ : variable of pressure $F=P^{2}$

$g$ : specific gravity

$h$ : bearing eccentric clearance $=C(1-\varepsilon \cos \theta)$

$h_{0}$ : dimensionless equilibrium bearing clearance $=\left(1-\varepsilon_{\mathrm{o}} \cos \theta_{\mathrm{o}}\right)$

$H$ : dimensionless bearing clearance $=h / C=(1-\varepsilon \cos \theta)$

$H_{w}$ : porous wall thickness

$i$ : imaginary number $=\sqrt{-1}$

$k$ : specific heat ratio of air $=\left(C_{p} / C_{v}\right)$

$K$ : permeability of porous bearing

$L$ : bearing length

$M$ : dimensionless threshold rotor mass supported per bearing= $\frac{\bar{M} C v^{2}}{2 \pi R L P_{a}}=\frac{W C v^{2}}{g}$

$\bar{M}$ : threshold rotor mass supported per bearing

$m$ : mesh number in $z$ direction $m=\frac{L}{2 \Delta z}$

$N$ : number of orifices in a circumferential row

$n$ : number of orifices per unit circumferential length $=N /(2 \pi R)$ $p$ : pressure

$P$ : dimensionless pressure $=p / p a$

$p_{a}$ : ambient pressure

$p_{o}$ : equilibrium pressure

$P_{o}$ : dimensionless equilibrium pressure $=p_{o} / P_{a}$

$P_{1}$ : dimensionless perturbed disturbance pressure $=p_{1} / p_{a}$

$P_{2}$ : dimensionless perturbed disturbance pressure $=p_{2} / p_{a}$

$p_{s}$ : supply pressure

$P_{s}:$ dimensionless supply pressure $=p_{s} / p_{a}$

$q_{x}, q_{y}$ : dimensionless displacements of journal center in $\mathrm{X}$ and $\mathrm{Y}$ coordinates $=\frac{\bar{q}_{x}}{C}, \frac{\bar{q}_{y}}{C}$

$\lambda_{o}$ : orifice feeding parameter $\lambda_{o}=\frac{24 \mu n a C_{d}\left(2 g \frac{k}{k-1}\right)^{\frac{1}{2}}}{p_{a^{\frac{1}{2}}} \rho_{a^{\frac{3}{2}}} C^{2}}$

$\lambda_{p}$ : porous feeding parameter $\lambda_{p}=\frac{12 K}{C H_{w}}$

$\omega$ : rotation frequency of journal $=U / R$

$v$ : whirl frequency of journal

$\gamma:$ whirl to rotation frequency ratio $=v / \omega$

$\tau$ : imaginary time $=i v t$

\section{Introduction}

The hybrid air journal bearings have been the important parts of the high-speed spindle for their cleanness and low friction of air lubrication. As the rotor supported by the bearings whirls during operation it may cause rotor dynamic instability. Thus the stiffness

*Corresponding author: K. N. Lie, Far East University, Tainan, 744, Taiwan ROC, E-mail: q6260@cc.feu.edu.tw

Received March 25, 2013; Accepted April 24, 2013; Published April 29, 2013

Citation: Lie KN, Su JCT (2013) A Numerical Analysis of Optimum Air Journal Bearings. J Appl Mech Eng 2: 122. doi:10.4172/2168-9873.1000122

Copyright: ( 2013 Lie KN, et al. This is an open-access article distributed under the terms of the Creative Commons Attribution License, which permits unrestricted use, distribution, and reproduction in any medium, provided the original author and source are credited. 
and damping coefficients of various designs of air bearings have been widely investigated by researchers for improving the problems of rotor dynamic instability.

The stability analysis of gas-lubricated hydrodynamic bearings has been the interest of researchers since the 1960's. The mathematical analyses of the whirl instability by Galerkin's method and by perturbation method were developed to investigate the threshold mass and threshold speed of rotor-supported bearings in the papers of Cheng and Trumpler [1], Cheng and Pan [2] and Castelli and Elord [3]. Subsequent investigations to improve the stiffness of gas bearings were carried out by Lund [4-5] in which the stiffness and damping coefficients were calculated to examine the whirl instabilities of 3-robe tilting pads and externally pressurized gas bearings respectively. Experimental investigation of hydrostatic gas journal bearings was also performed by Leonard and Rowe [6] in which the dynamic force coefficients and the mechanism of rotor whirling were investigated and the theoretical predictions of onset of instability by mathematical correlations were shown to be in good match with the experiments.

The porous bearings are also of interest to researchers for their better damping characteristics. Sun [7] investigated the hybrid instability of rotor whirling and pneumatic hammer effects in porous journal bearings. Subsequent studies, Wadhaw et al. [8], Han et al. [9] and Zhang and Chang [10], investigated externally pressurized air bearings numerically by various mathematical approaches for various configurations of compensation of pressurized air. Recent studies of rotor dynamic characteristics of both liquid and air bearings were conducted in the papers [11-17] and [18-20] respectively. Recently, by use of improved computing power, thermo-hydrodynamic lubrication is focused to obtain more precise analysis of the bearing lubrication in which the properties of lubricants are considered to be temperature dependent and the energy equation is included in the papers [21-22].

Although much effort has been made to investigate the mechanism of rotor-whirling instability of journal bearings, satisfactory results are not enough to interpret the interactive mechanism of rotor dynamic instability conclusively. Su and Lie [23-25] investigated the rotor dynamic instability of air journal bearings with new designs for compensation of pressurized air by multi-array of $1,2,3$, 4, or 5-row orifices and compares the stiffness and damping characteristics with the porous bearings for improving rotor-whirling instability. Otsu et al. [26] investigated pneumatic hammer instability of the aerostatic porous bearing and found that a surface-restricted layer with smaller permeability has good damping effect to avoid pneumatic hammer instability. Furthermore the present study investigates the responses to the changes of bearing length-to-diameter ratio and supply pressure of the above new-designed hybrid air journal bearings with rotor dynamic instability for more insight in design of more stable bearings.

\section{Mathematical Analysis}

The schematic illustrations of journal bearings with multi-array of $1,2,3,4$, or 5-row orifice feedings and porous feeding are shown in figure 1 . The supply of pressurized air is restricted by the orifices in the orifice compensated bearings or by the porous matrix in the porous bearings. The orifices considered here could be stepped holes with thin needle throttle holes to discharge pressurized air to the lubricating film. A line source was used in the simulations so that predictions are valid for bearings having eight or more orifices in each circumference. The porous matrix in the porous bearings considered here could be a sintered material with porosity lower than $10^{-10} \mathrm{~cm}^{2}$. It should be noted

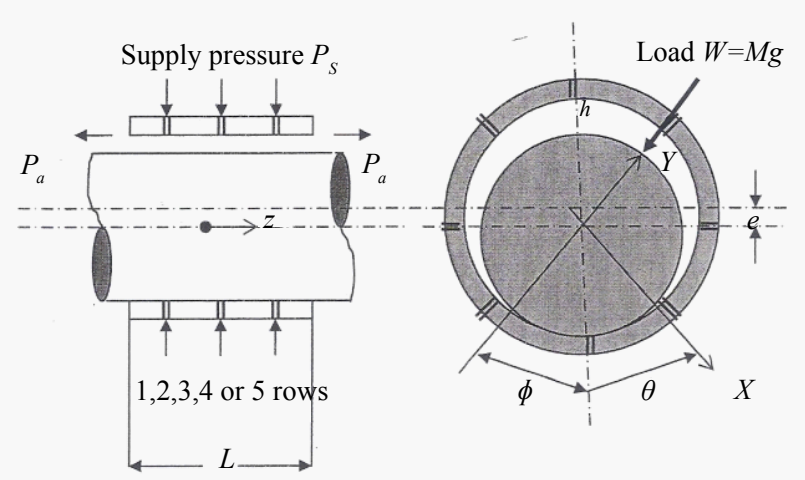

(a) Air journal bearing of multi-array of orifice feedings

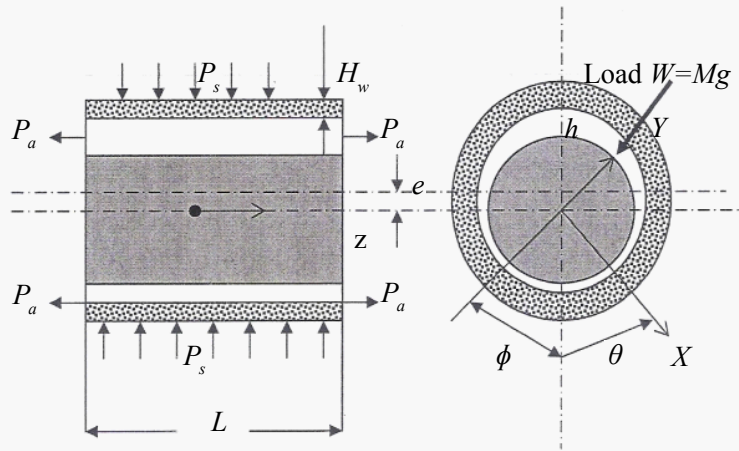

(b) Air journal bearing of porous feeding.

Figure 1: Illustrations of (a) multi-array of orifice (b) porous journal bearings.

that one to five rows of orifices are considered in the current study and that the rows are spaced equally on the axial cross sections from both bearing sides and arrayed symmetrically to the center cross section of the bearing with $1,2,3,4$ or 5 circumferential rows. A thin lubricating film and the compressibility of air like an ideal gas are considered in the analysis. The Darcy flow through porous wall in the porous bearing is assumed. The Reynolds equation which is only valid for low Reynolds numbers of order of unity is applicable for a thin lubricating film of order of $0.1 \mathrm{~mm}$ in a bearing. The dimensionless governing Reynolds equations for a journal bearing with rotation and whirl can be written as:

(A) Orifice feeding

$\frac{\partial}{\partial \theta}\left(H^{3} \frac{\partial P^{2}}{\partial \theta}\right)+\left(\frac{D}{L}\right)^{2} \frac{\partial}{\partial Z}\left(H^{3} \frac{\partial P^{2}}{\partial Z}\right)=2 \Lambda \frac{\partial(P H)}{\partial \theta}+i 4 \gamma \Lambda \frac{\partial(P H)}{\partial \tau}$

(B) Porous feeding

$\frac{\partial}{\partial \theta}\left(H^{3} \frac{\partial P^{2}}{\partial \theta}\right)+\left(\frac{D}{L}\right)^{2} \frac{\partial}{\partial Z}\left(H^{3} \frac{\partial P^{2}}{\partial Z}\right)=2 \Lambda \frac{\partial(P H)}{\partial \theta}-\frac{24 \mu K R^{2}}{C^{3}}\left(P P_{s}-P^{2}\right)+i 4 \gamma \Lambda \frac{\partial(P H)}{\partial \tau}$

where the additional term in (2) as compared to (1) comes from the Darcy flow velocity $V_{p}$ in the radial direction through the porous bearing to the lubricating air film.

If the journal positions are perturbed by $\varepsilon_{1}$ and $\phi_{1}$ from their static equilibrium positions identified by eccentricity ratio $\varepsilon_{0}$ and attitude angle $\phi_{0}$, the new positions are given by 


$$
\varepsilon=\varepsilon_{0}+\varepsilon_{1}, \quad \phi=\phi_{0}+\phi_{1}
$$

Here $\varepsilon_{1}$ and $\phi_{1}$ are subjected to a small whirling harmonic motion and can be defined as:

$$
\varepsilon_{1}=\operatorname{Re}\left\{\left|\varepsilon_{1}\right| e^{\tau}\right\}, \quad \phi_{1}=\operatorname{Re}\left\{\left|\phi_{1}\right| e^{\tau}\right\}
$$

The corresponding equations for the dimensionless film thickness and pressure in the perturbed state are

$$
\begin{aligned}
& H=h_{0}-\varepsilon_{1} \cos \theta_{0}+\varepsilon_{0} \phi_{1} \sin \theta_{0} \\
& P=P_{0}+\varepsilon_{1} P_{1}+\varepsilon_{0} \phi_{1} P_{2}
\end{aligned}
$$

where $h_{0}$ is the dimensionless equilibrium film thickness and expressed as:

$$
h_{0}=1+\varepsilon_{0} \cos \theta_{0}
$$

Substituting the perturbed quantities of (4-6) into the governing Reynolds equations (1-2), the perturbed governing equations are formulated for orifice feeding and porous feeding bearings to be (A1) and (B1) respectively as shown in appendices A and B. In derivations of (A1) and (B1), higher order perturbed terms than $\varepsilon_{1}$ and $\phi_{1}$ are ignored. In order to solve the disturbance quantities of pressures $\mathrm{P} 1$ and $\mathrm{P} 2,(\mathrm{~A} 1)$ and (B1) are differentiated partially with respect to $\varepsilon_{1}$ and $\varepsilon_{0} \phi_{1}$ and result in (A2), (A3), (B2) and (B3) in appendices A and $\mathrm{B}$. The three common boundary conditions for the perturbed equations (A2), (A3), (B2) and (B3) for orifice and porous feedings are defined as following:

$$
\begin{aligned}
& P_{1}(\theta, Z=1)=P_{2}(\theta, Z=1)=0 \\
& P_{1}(\theta, Z)=P_{1}(\theta+2 \pi, Z), \quad P_{2}(\theta, Z)=P_{2}(\theta+2 \pi, Z) \\
& \frac{\partial P_{1}}{\partial \theta}(\theta, Z)=\frac{\partial P_{1}}{\partial \theta}(\theta+2 \pi, Z), \quad \frac{\partial P_{2}}{\partial \theta}(\theta, Z)=\frac{\partial P_{2}}{\partial \theta}(\theta+2 \pi, Z)
\end{aligned}
$$

The continuity conditions at orifices are needed to solve the perturbed equations (A2), (A3), (B2) and (B3). The continuity equation in the locations of orifice inlets is written as:

$$
\frac{\partial}{\partial x}\left(\frac{\rho h^{3}}{\mu} \frac{\partial P}{\partial x}\right)+\frac{\partial}{\partial z}\left(\frac{\rho h^{3}}{\mu} \frac{\partial P}{\partial z}\right)=6 U \frac{\partial(\rho h)}{\partial x}+12 \frac{\partial(\rho h)}{\partial t}-12 \rho v_{o}
$$

where $v_{o}$ is the discharge velocity of air through the orifice. The formulations of $v_{o}$ are shown as in Su and Lie [24]. The dimensionless form of (11) is written as:

$$
\frac{\partial}{\partial \theta}\left(H^{3} \frac{\partial P^{2}}{\partial \theta}\right)+\left(\frac{D}{L}\right)^{2} \frac{\partial}{\partial Z}\left(H^{3} \frac{\partial P^{2}}{\partial Z}\right)=2 \Lambda \frac{\partial(P H)}{\partial \theta}+i 4 \gamma \Lambda \frac{\partial(P H)}{\partial \tau}-4 \Lambda V_{0}
$$

The final perturbed forms of the continuity (12) are derived to be (A4) and (A5) in appendix A.

The fourth boundary condition for the porous bearing is the symmetric condition in the center cross section of the porous bearing and expressed as:

$$
\frac{\partial P_{1}}{\partial Z}(\theta, Z=0)=\frac{\partial P_{2}}{\partial Z}(\theta, Z=0)=0
$$

\section{Numerical solutions for perturbed equations}

The central finite difference method is employed to solve the perturbed equations (A2) and (A3) with boundary conditions of (8-10), (A4) and (A5) for orifice feeding bearings, and the perturbed equations (B2) and (B3) with boundary conditions of $(8-10,13)$ are also solved for porous bearings. The equilibrium pressure $P_{o}$ is to be solved with a set of fixed parameters for a certain operating condition of the flow in the bearing and then inserted into the perturbed equations to solve the disturbance pressures $P_{1}$ and $P_{2}$ for the disturbed flow in the bearing. The solution of the equilibrium pressure $P_{0}$ can be referred to $\mathrm{Su}$ and Lie [24]. It should be noted that double precision in programming is required for the disturbance pressures $P_{1}$ and $P_{2}$ to improve the numerical convergence. A mesh number of $30 \times 180$ in radial and angular directions makes the load capacity of the bearing converge to a relative error within $10^{-5}$ and is employed in the numerical schemes. The criterion of numerical iterating convergence is set to be that the accumulated relative error of all grids between two iterations is less than $10^{-5}$ :

$$
\sum_{i} \sum_{j}\left|\frac{P_{i, j}^{n+1}-P_{i, j}^{n}}{P_{i, j}^{n+1}}\right|<10^{-5}
$$

\section{Calculation of stiffness and damping coefficients}

The perturbed support force components $F_{R}$ along and $F_{T}$ perpendicular to the line of centers of journal and bearing can be determined as follows:

$$
\begin{aligned}
& F_{R}=F_{R 0}+Z_{R R} \varepsilon_{1}+Z_{R T} \varepsilon_{0} \phi_{1} \\
& F_{T}=F_{T 0}+Z_{T R} \varepsilon_{1}+Z_{T T} \varepsilon_{0} \phi_{1}
\end{aligned}
$$

where $F_{R O}$ and $F_{T O}$ are the equilibrium support force components and determined as Su and Lie (24). And the perturbed force coefficients are determined by integration as:

$$
\begin{gathered}
\left\{\begin{array}{l}
Z_{R R} \\
Z_{T R}
\end{array}\right\}=2 \int_{0}^{1} \int_{0}^{2 \pi} P_{1}\left\{\begin{array}{c}
\cos \theta \\
\sin \theta
\end{array}\right\} d \theta d Z \\
\left\{\begin{array}{l}
Z_{R T} \\
Z_{T T}
\end{array}\right\}=2 \int_{0}^{1} \int_{0}^{2 \pi} P_{2}\left\{\begin{array}{c}
\cos \theta \\
\sin \theta
\end{array}\right\} d \theta d Z
\end{gathered}
$$

The perturbed force coefficients for the $X$ and $Y$ components are expressed in the following matrix transformation:

$$
\left[\begin{array}{ll}
Z_{X X} & Z_{X Y} \\
Z_{Y X} & Z_{Y Y}
\end{array}\right]=\left[\begin{array}{ll}
Z_{R R} & Z_{T R} \\
Z_{R T} & Z_{T T}
\end{array}\right]\left[\begin{array}{cc}
\sin \phi_{o} & -\cos \phi_{o} \\
\cos \phi_{o} & \sin \phi_{o}
\end{array}\right]\left[\begin{array}{cc}
\sin \phi_{o} & -\cos \phi_{o} \\
\cos \phi_{o} & \sin \phi_{o}
\end{array}\right]
$$

The perturbed force coefficients are complex numbers and can be separated in real and imaginary matrixes as:

$$
\left[\begin{array}{ll}
Z_{X X} & Z_{X Y} \\
Z_{Y X} & Z_{Y Y}
\end{array}\right]=\left[\begin{array}{ll}
S_{11} & S_{12} \\
S_{21} & S_{22}
\end{array}\right]+i\left[\begin{array}{ll}
B_{11} & B_{12} \\
B_{21} & B_{22}
\end{array}\right]
$$

where $S_{11}, S_{12}, S_{21}$ and $S_{22}$ are the stiffness coefficients and $B_{11}, B_{12}, B_{21}$ and $B_{22}$ are the damping coefficients of the journal bearings.

\section{Whirl instability analysis}

The whirl motion $q$ of rotor mass $M$ can be written in linear differential equations as:

$$
\left[\begin{array}{cc}
M & 0 \\
0 & M
\end{array}\right]\{\ddot{q}\}+\left[\begin{array}{ll}
B_{11} & B_{12} \\
B_{21} & B_{22}
\end{array}\right]\{\dot{q}\}+\left[\begin{array}{ll}
S_{11} & S_{12} \\
S_{21} & S_{22}
\end{array}\right]\{q\}=\left\{\begin{array}{l}
0 \\
0
\end{array}\right\}(21)
$$

where $q$ is a two dimensional vector of displacements of $q_{x}$ and $q_{y}$ in $X$ and $Y$ directions respectively.

The threshold mass $M$ of rotor is determined by use of Routh's criterion. The weight of rotor mass is accounted as the entire loading of the bearing $(W=M g)$ and balanced by the equilibrium support forces $F_{R 0}$ and $F_{T 0}$ of the bearing.

By applying Routh's criterion, the standard of whirl instability can 
be derived from the (21) of motion as:

$$
\left[\begin{array}{cc}
M+B_{11}+S_{11} & B_{12}+S_{12} \\
B_{21}+S_{21} & M+B_{22}+S_{22}
\end{array}\right]\left\{\begin{array}{l}
q_{x} \\
q_{y}
\end{array}\right\}=\left\{\begin{array}{l}
0 \\
0
\end{array}\right\}
$$

The neutral stability criterion for existence of solution of (22) requires the coefficient determinant to be zero:

$$
\left|\begin{array}{cc}
M+B_{11}+S_{11} & B_{12}+S_{12} \\
B_{21}+S_{21} & M+B_{22}+S_{22}
\end{array}\right|=0
$$

The iteration procedure with Newton-Raphson correcting algorithm is employed to determine the threshold rotor mass $M$ by correcting the values of whirl to rotation frequency ratio $\gamma$ under a certain set of fixed operation parameters that define an equilibrium flow in a bearing. The convergence criterion is set for the residue of the determinant (23) to be less than $10^{-5}$.

\section{Results and Discussions}

The responses to changes of bearing length-to-diameter ratio and supply pressure of hybrid air journal bearings are analyzed numerically for the whirl instability of rotor mass. The various external pressure compensations of air, including the bearing of multi-array of $1,2,3$, 4 , or 5 circumferential rows of orifice feeding and the porous bearing, are analyzed to obtain more information for the optimum designs. The threshold rotor mass $\bar{M}$, which constitutes the load capacity $w(=\bar{M}$ g) of supporting bearings, is defined as the measure of whirl instability.

Figure 2 shows threshold load capacity $W$ of rotor mass vs. bearing length to radius ratio $(L / D)$ for orifice feeding parameter $\lambda_{o}=10^{-4}$ and porous feeding parameter $\lambda_{p}=10^{-8}$ for bearing number $\Lambda=0.1$. It is seen that the threshold load capacity $\mathrm{W}$ increases with bearing length to radius ratio $(L / D)$ almost linearly in a lower rotation speed $(\Lambda=0.1)$. It can be seen that 5-row orifice bearing has the higher threshold load capacity $W$ and thus is more stable than the porous bearing in a lower rotation speed.

Figure 3 shows threshold load capacity $W$ of rotor mass vs. bearing length to radius ratio $(L / D)$ for orifice feeding parameter $\lambda_{o}=10^{-4}$ and

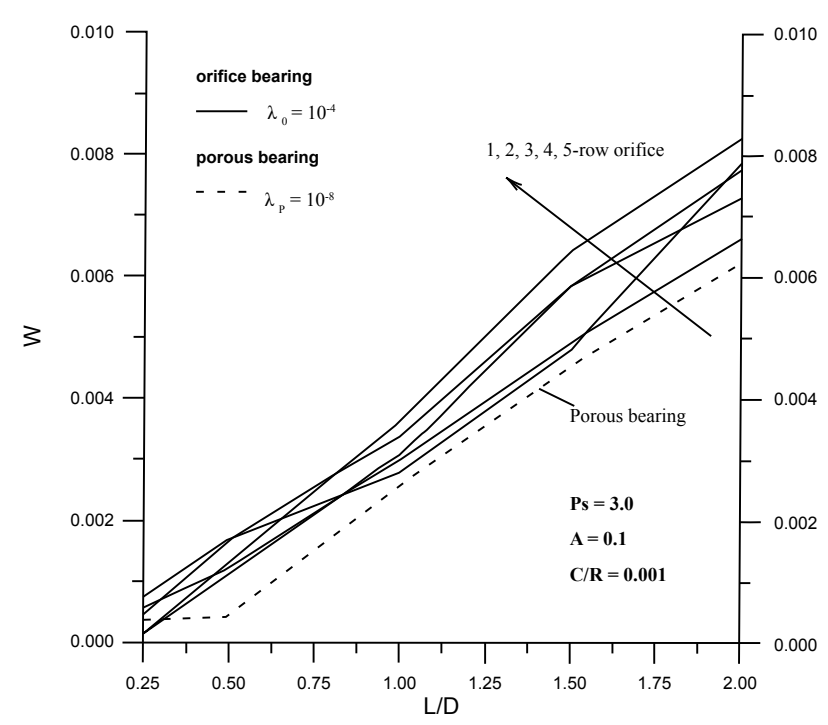

Figure 2: Threshold load capacity $W$ of rotor mass vs. bearing length to radius ratio $(L / D)$.

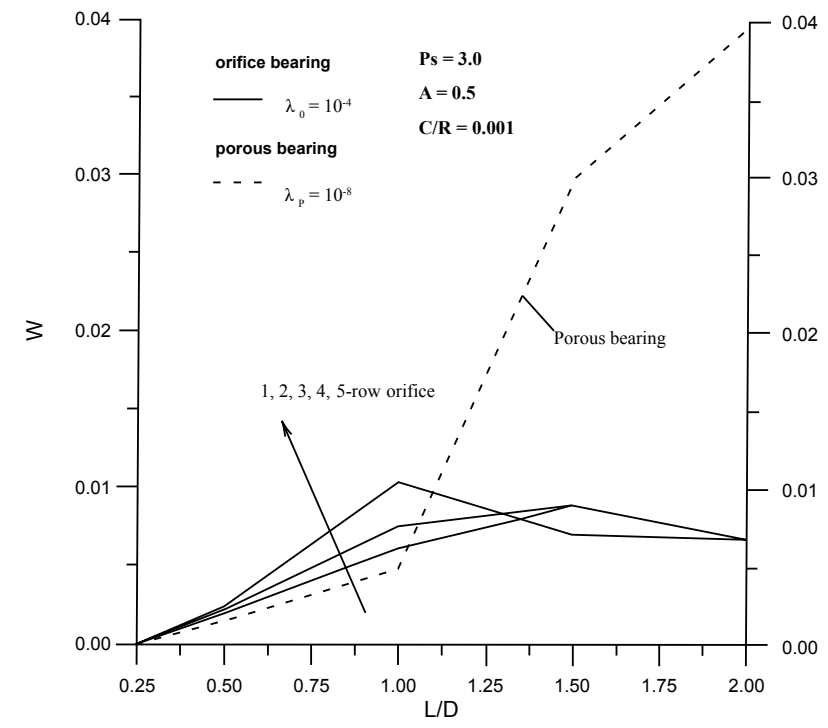

Figure 3: Threshold load capacity $W$ of rotor mass vs. bearing length to radius ratio $(L / D)$.

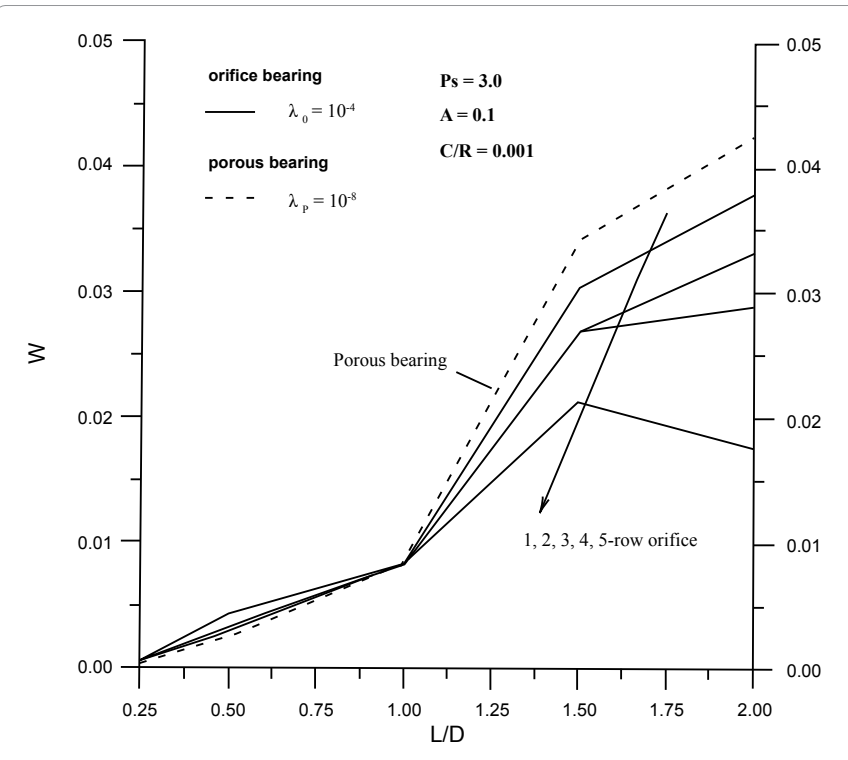

Figure 4: Threshold load capacity $W$ of rotor mass vs. bearing length to radius ratio $(L / D)$.

porous feeding parameter $\lambda_{p}=10^{-8}$ for bearing number $\Lambda=0.5$. It is seen that the threshold load capacity $W$ of the porous bearing increases steeply as the ratio $(L / D)$ exceeds 1.0. It is apparent that the long porous bearing $(L / D>1)$ is more stable in a medium rotation speed $(\Lambda=0.5)$. It can be shown that the stiffness of a long porous bearing decrease as the slenderness ratio $(L / D)$ exceeds 1.0 as in (23) and that the damping coefficient of a porous bearing is higher than an orifice bearing as in (24). Both low stiffness and high damping effect make a long porous bearing more stable. It can also be seen that 5-row orifice bearing has the higher threshold load capacity $W$ and then is more stable for short bearings $(L / D \leqq 1)$ in a medium rotation speed $(\Lambda=0.5)$.

Figure 4 shows threshold load capacity $W$ of rotor mass vs. bearing length to radius ratio $(L / D)$ for orifice feeding parameter $\lambda_{0}=10^{-4}$ and porous feeding parameter $\lambda_{p}=10^{-8}$ for bearing number $\Lambda=1.0$. It is seen 


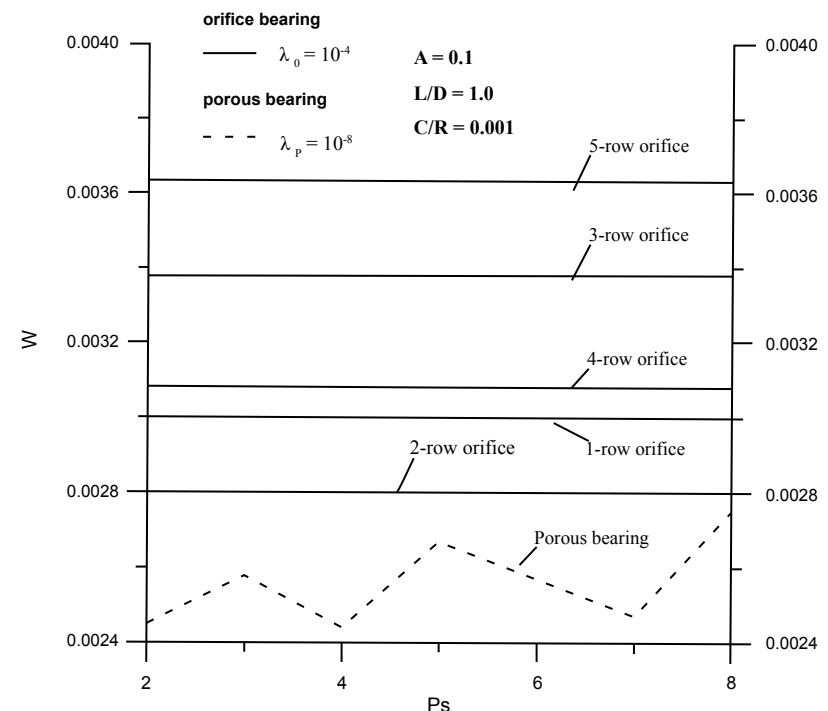

Figure 5: Threshold load capacity $W$ of rotor mass vs. supply pressure $P_{\mathrm{s}}$

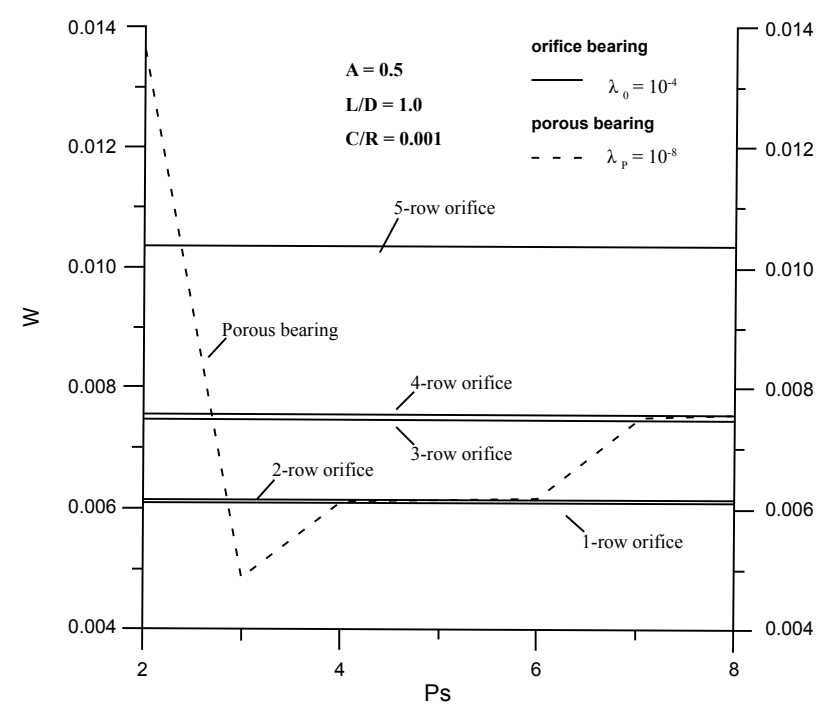

Figure 6: Threshold load capacity $W$ of rotor mass vs. supply pressure $P_{\mathrm{s}} 5$.

that the threshold load capacities $W$ of all types of bearings increase similarly with the ratio $(L / D)$ until $L / D=1.5$ and that the threshold load capacity $W$ of 5 -row orifice bearing decreases as the ratio $(L / D)$ exceeds 1.5 in a higher rotation speed $(\Lambda=1.0)$. It can be seen that there is no significant difference with the threshold load capacities $W$ of all types of bearings for short bearings $(L / D \leqq 1)$ in a higher rotation speed $(\Lambda=1.0)$. It can also be seen that the long porous bearing $(L / D>1)$ has higher threshold load capacity $W$ and thus is more stable than the orifice bearings in a higher rotation speed $(\Lambda=1.0)$ for the same reason that the stiffness of a long porous bearing decreases steeply as slenderness exceeds $1(L / D>1)$ as shown in (23).

Figure 5 shows threshold load capacity $W$ of rotor mass vs. supply pressure $P_{s}$ for orifice feeding parameter $\lambda_{0}=10^{-4}$ and porous feeding parameter $\lambda_{p}=10^{-8}$ for bearing number $\Lambda=0.1$. It is seen that the threshold load capacities $W$ of orifice bearings have no change as supply pressure increases from $P_{s}=2.0$ to $P_{s}=8.0$ and that the threshold load capacity $W$ of the porous bearing has no significant change in average in a lower rotation speed $(\Lambda=0.1)$. It is also seen that the porous bearing has the lower threshold load capacities than the orifice bearings for the porous bearing has the higher stiffness than the orifice bearings in a low rotation speed. It is also seen that the odd-row orifice bearings have the higher threshold load capacities and then are stabilized by locating a row in the bearing center cross section.

Figure 6 shows threshold load capacity $W$ of rotor mass vs. supply pressure Ps for orifice feeding parameter $\lambda=10^{-4}$ and porous feeding parameter $\lambda_{p}=10^{-8}$ for bearing number $\Lambda=0.5$. It is seen that the porous bearing has the higher threshold load capacity $W$ for the lower supply pressure $P_{s}=2.0$ and the threshold load capacities $W$ of orifice bearings have no change as supply pressure increases from $P_{s}=2.0$ to $P_{s}=8.0$ in a medium rotation speed $(\Lambda=0.5)$. The more stable porous bearing at low pressure $P_{s}=2.0$ comes out with its low stiffness and high damping effect.

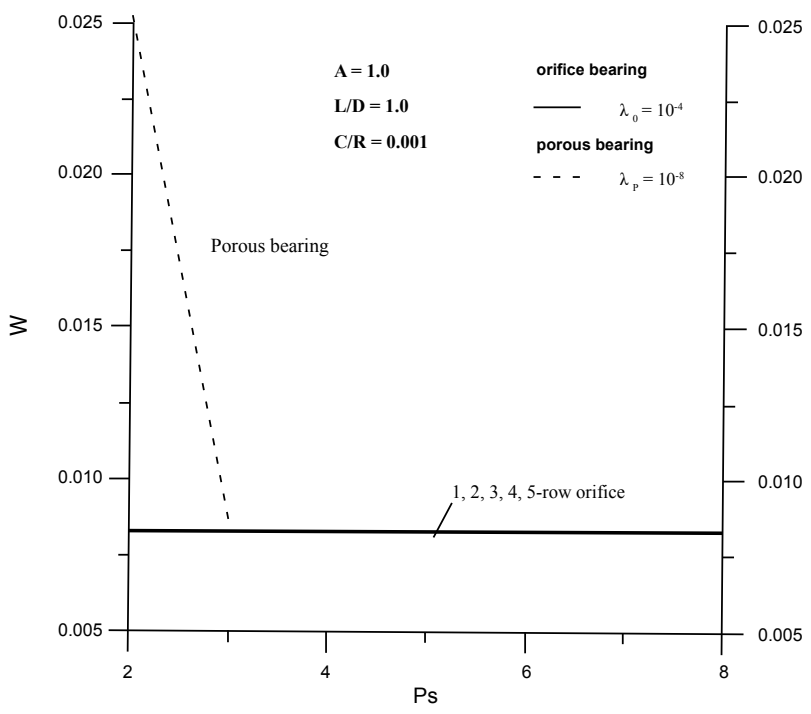

Figure 7: Threshold load capacity $W$ of rotor mass vs. supply pressure $P_{\mathrm{s}}$

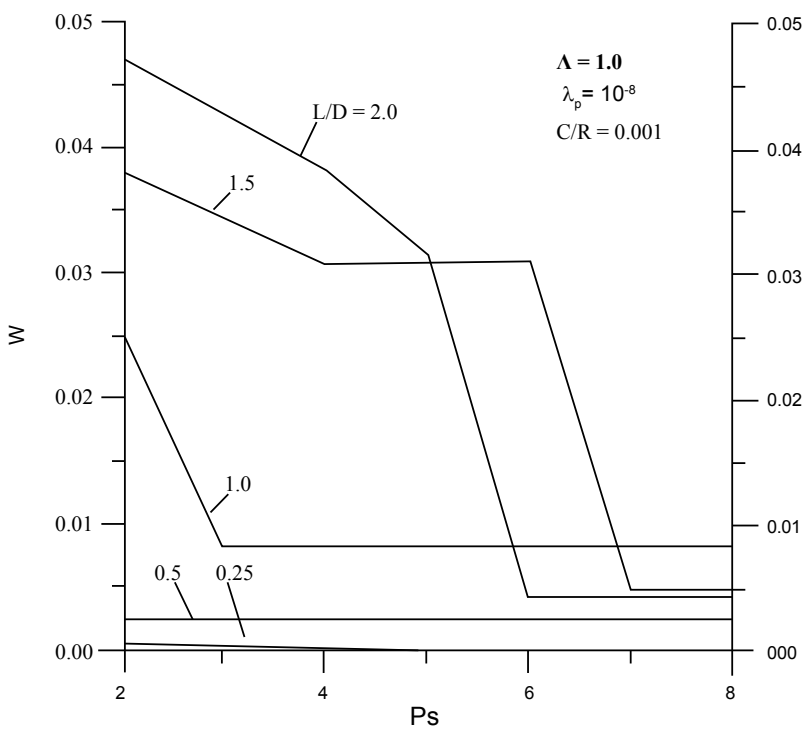

Figure 8: Threshold load capacity $W$ of rotor mass vs. supply pressure $P_{\mathrm{s}}$ 


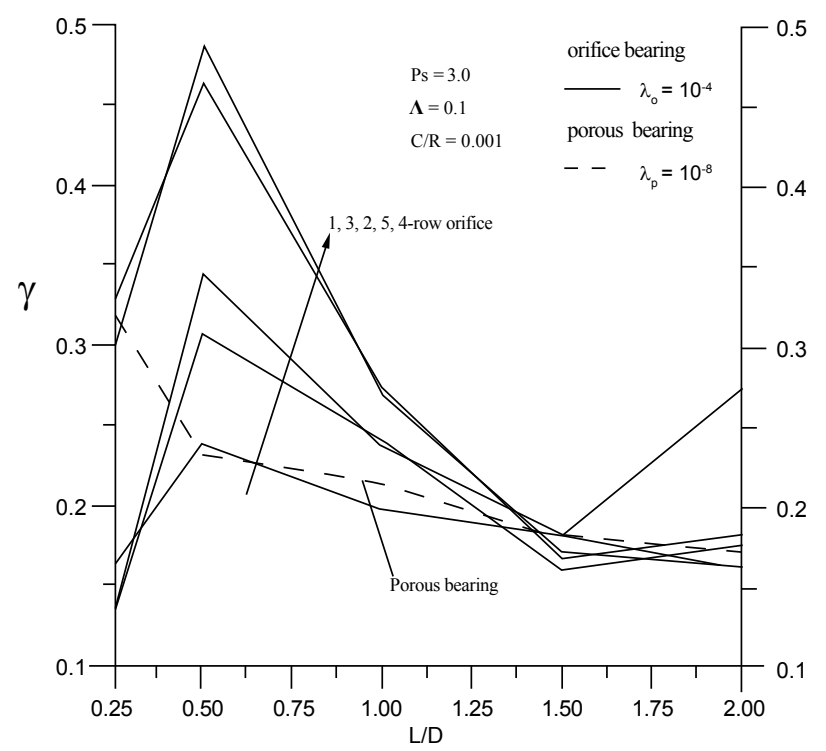

Figure 9: Threshold whirl frequency ratio $y$ vs. bearing length to radius ratio $(L / D)$.

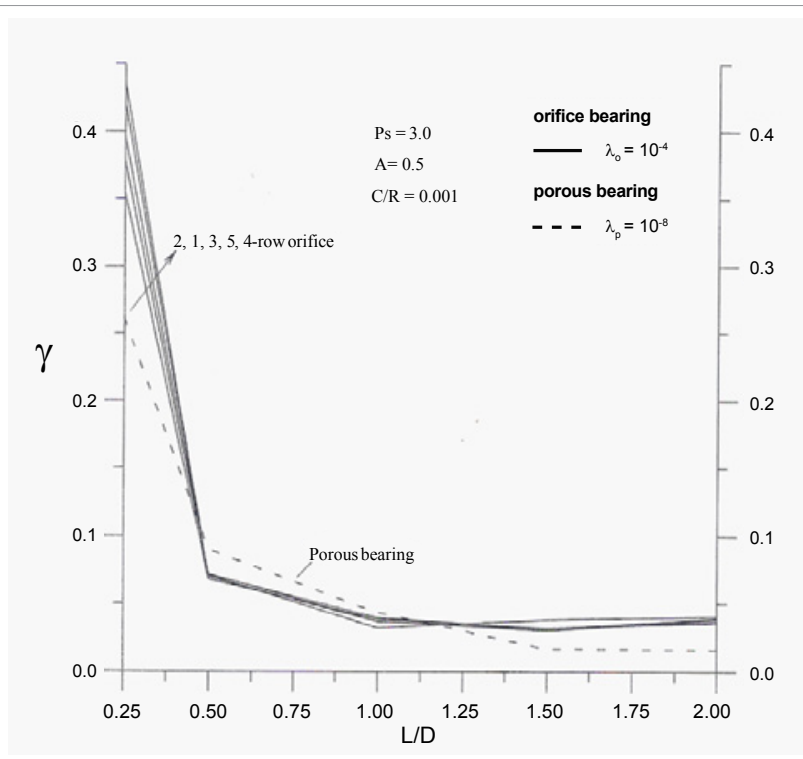

Figure 10: Threshold whirl frequency ratio $y$ vs. bearing length to radius ratio $(L / D)$.

Figure 7 shows threshold load capacity $\mathrm{W}$ of rotor mass vs. supply pressure Ps for orifice feeding parameter $\lambda_{\mathrm{o}}=10-4$ and porous feeding parameter $\lambda p=10-8$ for bearing number $\Lambda=1.0$. It is seen that the threshold load capacities $\mathrm{W}$ are almost the same as supply pressure increases from $\mathrm{Ps}=2.0$ to $\mathrm{Ps}=8.0$ except that the porous bearing has the higher threshold load capacity $\mathrm{W}$ for the lower supply pressure $\mathrm{Ps}=2.0$ in a higher rotation speed $(\Lambda=1.0)$. It is apparent from figures 6 and 7 that the porous bearing has the higher threshold load capacity $\mathrm{W}$ for lower supply pressure Ps=2.0 and thus is more stable in the higher rotation speeds $(\Lambda \geqq 0.5)$ by its low stiffness and high damping effect at the lower supply pressure $P=2.0$. It can be considered that the limitation of the critical pressure ratio $\left(P_{o} / P_{s}=0.53\right)$ of air flowing through an orifice causes no substantial change is observed with rotor instability as supply pressure $P_{s}$ increases from 2.0 to 8.0 .
Figure 8 shows threshold load capacity $W$ of rotor mass vs. supply pressure $P$ for various bearing length to radius ratio $(L / D)$ for porous bearings for porous feeding parameter $\lambda_{p}^{s}=10^{-8}$ for bearing number $\Lambda=1.0$. It is seen that the long porous bearing $(L / D \geqq 1)$ has the higher threshold load capacity $W$ for the lower supply pressure $P_{s}=2.0$ in a higher rotation speed $(\Lambda=1.0)$. It can be said that the porous bearing is relatively stable for lower supply pressures and becomes unstable rapidly as supply pressure increases. This is because the stiffness of the porous bearing increases substantially as the supply pressure $P_{s}$ increases and the higher stiffness would degrade the stability of rotor whirl. But the orifice bearing differs from the porous bearing for its stiffness does not increase significantly as the supply pressure $P_{s}$ increases because of the limitation of critical pressure ratio $\left(P_{o} / P_{s}=0.53\right)$ of air flowing through an orifice. The stiffness of an orifice feeding bearing no longer increases as the supply pressure ratio exceeds the critical pressure ratio. Therefore the threshold load capacities $W$ of orifice bearings have no significant change as supply pressure increases from $P_{s}=2.0$ to $P_{s}=8.0$ as shown in figures 5-7.

Figure 9 shows threshold whirl frequency ratio $\gamma$ vs. bearing length to radius ratio $(L / D)$ for orifice feeding parameter $\lambda_{0}=10^{-4}$ and porous feeding parameter $\lambda_{p}=10^{-8}$ for bearing number $\Lambda=0.1$. It can be seen that the 5-row and 4-row orifice bearings have relatively higher threshold whirl frequency ratios $\gamma$ than the porous bearing in average in a lower rotation speed $(\Lambda=0.1)$. In addition, the 5-row and 4-row orifice bearings have relatively higher threshold load capacity $W$ than the porous bearing as shown in figure 2. It is concluded that the 5-row and 4-row orifice bearings are more stable than the porous bearing in a lower rotation speed $(\Lambda=0.1)$.

Figure 10 shows threshold whirl frequency ratio $\gamma$ vs. bearing length to radius ratio $(L / D)$ for orifice feeding parameter $\lambda_{0}=10^{-4}$ and porous feeding parameter $\lambda=10^{-8}$ for bearing number $\Lambda=0.5$. It is seen that all the threshold whirl frequency ratio $\gamma$ are close to each other as the ratio $(L / D)$ exceeds 0.5 and the whirl frequency ratio $\gamma$ of 5-row and 4-row orifice bearings are higher than that of the porous bearing when the ratio $(L / D)$ is less than 0.5 . As referred to figure 3 , it can be seen that the 5-row orifice bearing has the higher threshold load capacity $W$ with the higher whirl frequency ratio $\gamma$ and thus is more stable than the porous bearing for a short bearing $(L / D \leqq 1)$ in a medium rotation

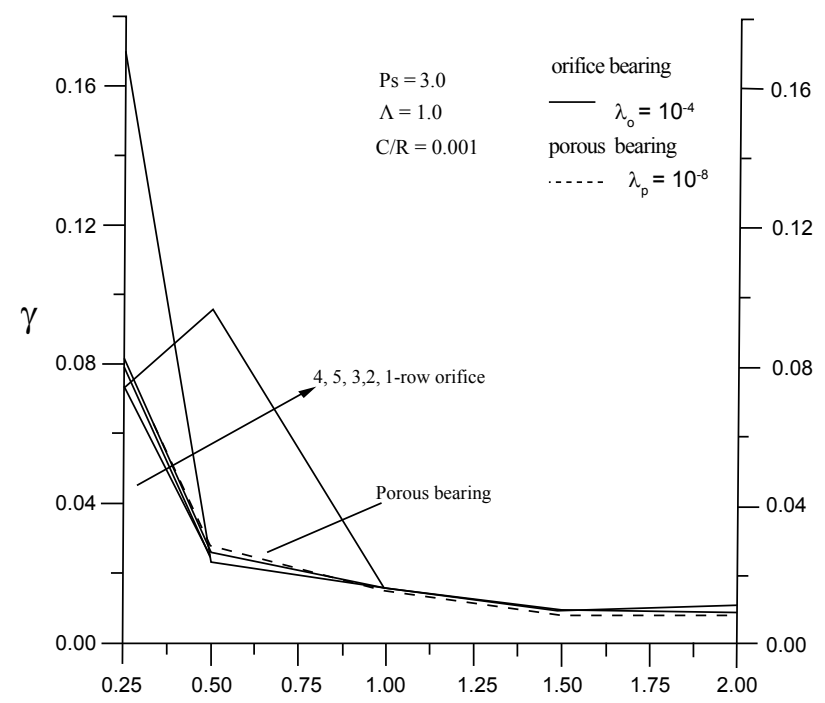

Figure 11: Threshold whirl frequency ratio $y$ vs. bearing length to radius ratio $(L / D)$. 


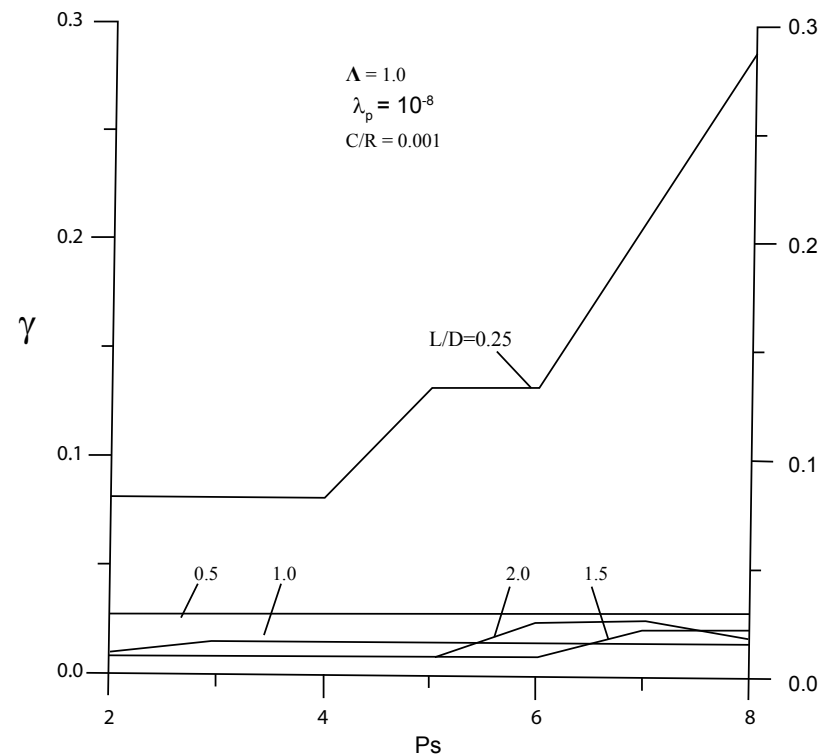

Figure 12: Threshold whirl frequency ratio $Y$ vs. supply pressure $P_{s}$.

speed $(\Lambda=0.5)$ and that the long porous bearing $(L / D>1)$ has much higher threshold load capacity $W$ with a little lower whirl frequency ratio $\gamma$ and thus is more stable than orifice bearings in a medium rotation speed $(\Lambda=0.5)$.

Figure 11 shows threshold whirl frequency ratio $\gamma$ vs. bearing length to radius ratio $(L / D)$ for orifice feeding parameter $\lambda_{o}=10^{-4}$ and porous feeding parameter $\lambda=10^{-8}$ for bearing number $\Lambda=1.0^{\circ}$. It is seen that all the threshold whirl frequency ratio $\gamma$ are close to each other as the ratio $(L / D)$ exceeds 1.0 and the whirl frequency ratio $\gamma$ of 1-row and 2 -row orifice bearings are higher than that of the porous bearing when the ratio $(L / D)$ is less than 1.0 .

As referred to figure 4, It can be seen that the 1-row and 2-row orifice bearing has both higher threshold load capacity $W$ with the higher whirl frequency ratio $\gamma$ and thus are more stable than the porous bearing for a short bearing $(L / D \leqq 1)$ in a higher rotation speed $(\Lambda=1.0)$ but that the long porous bearing $(L / D>1)$ has much higher threshold load capacity $W$ with a little lower whirl frequency ratio $\gamma$ and thus is more stable than orifice bearings in a higher rotation speed $(\Lambda=1.0)$.

Figure 12 shows threshold whirl frequency ratio $\gamma$ vs. supply pressure $P_{s}$ for various bearing length to radius ratio $(L / D)$ for porous bearings for porous feeding parameter $\lambda_{p}=10^{-8}$ for bearing number $\Lambda=1.0$. It can be seen that the short porous bearing $(L / D=0.25)$ has higher threshold whirl frequency ratio $\gamma$ but much lower threshold load capacity $W$ as referred to figure 8 , so that the short porous bearing is unstable even with the higher threshold whirl frequency ratio $\gamma$.

It can also be seen that the long porous bearing $(L / D \geqq 1)$ has lower threshold whirl frequency ratio $\gamma$ but much higher threshold load capacity $W$ as referred to figure 8 for the lower supply pressure $P_{s}=2.0$ for bearing number $\Lambda=1.0$. Thus it can be said that the long porous bearing $(L / D \geqq 1)$ is more stable for the lower supply pressure $P_{s}=2.0$ in a higher rotation speed $(\Lambda=1.0)$.

\section{Conclusions}

Comparing multi-array orifice feeding air journal bearings with porous air journal bearings, some conclusions for the responses to changes of bearing length-to-diameter ratio and supply pressure with rotor whirl instability of hybrid air journal bearings are obtained as follows:

1. A long bearing is more stable than a short bearing generally for the threshold load capacity increases with the bearing length to radius ratio $(L / D)$.

2. The 5-row orifice bearing is more stable than the porous bearing for a short bearing $(L / D \leqq 1)$ and in the lower rotation speed $(0.1 \leqq \Lambda$ $\leqq 0.5)$.

3. The porous bearing is more stable than the orifice bearings for a long bearing $(L / D>1)$ in the higher rotation speed $(\Lambda>0.5)$.

4. The orifice bearings are insensible to the change of external supply pressure with rotor whirl instability and their threshold load capacities of rotor mass have no significant change as supply pressure increases from $P_{s}=2.0$ to $P_{s}=8.0$.

5. The long porous bearings $(L / D \geqq 1)$ is more stable for the lower supply pressure $P_{s}=2.0$ for the threshold load capacity drops rapidly as the supply pressure increases from the lower $P_{s}=2.0$ for the long porous bearings.

\section{Acknowledgment}

It is our acknowledgment to National Science Council of R.O.C. for its financial support for the research project NSC88-2622-E-327-001 "Design and Manufacture of High Speed Air Spindle" and to National Kaohsiung First University of Science and Technology for use of its facilities in this project.

\section{References}

1. Cheng HS, Trumpler PR (1963) Stability of High-Speed Journal Bearing under Steady Load. J Manuf Sci Eng 85: 274-279.

2. Cheng HS, Pan C H T (1963) Stability Analysis of Gas-Lubricated, Self-Acting Plain, Cylindrical. Journal Bearings of Finite Length, Using Galerkin's Method. ASME J Basic Engineering 185-192.

3. Castelli V, Elord H G (1963) Solution of the Stability Problem for 360 Deg. SelfActing, Gas-Lubricated Bearings. ASME J Basic Engineering 199-211.

4. Lund JW (1965) A Theoretical Analysis of Whirl Instability and Pneumatic Hammer for a Rigid Rotor in Pressurized Gas Journal Bearings. ASME J Lubri Tech 154-165.

5. Lund JW (1968) Calculation of Stiffness and Damping Properties of Gas Bearings. ASME J Lubri Tech 793-803.

6. Leonard R, Rowe WB (1973) Dynamic Force Coefficients and the Mechanism of Instability in Hydrostatic Journal Bearings. Wear 23: 277-282.

7. Sun DC (1975) Stability of Gas-Lubricated, Externally Pressurized Porous Journal Bearings. ASME J Lubri Tech 494-505

8. Wadhwa SS, Sinhasan R, Singh DV (1983) Analysis of Orifice Compensated Externally Pressurized Gas Bearings, Tribol Int 16: 203-211.

9. Han DC, Park SS, Kim JW (1994) A Study on The Characteristics of Externally Pressurized Air Bearings. Precision Engineering 16: 164-173.

10. Zhang RQ, Chang HS (1995) A New Type of Hydrostatic/Hydrodynamic Gas Journal Bearing and Its Optimization for Maximum Stability. Tribol Trans 38: 589-594.

11. WB Rowe (1980) Dynamic and Static Properties of Recessed Hydrostatic Journal Bearings by Small Displacement Analysis. ASME J Lubri Tech 102 : 71-79.

12. Abdul-Wahed ND, Nicolas MT, Pascal (1982) Stability and Unbalance Response of Large Turbine Bearings. ASME J Lubri Tech 104: 66-75.

13. Chen YS, Wu HY, Xie PL, (1985) Stability of Multirecess Hybrid-Operating Oil Journal Bearings. ASME J Tribol 107: 116-121.

14. Kocu JA, Allaire PE (1986) Finite Element Analysis of Turbulent Lubricated Hydrostatic Journal Bearings for Static and Dynamic Conditions. ASLE Trans 29: 126-135. 
15. Andres LS (1990) Approximate Analysis of Turbulent Hybrid Bearings, Static and Dynamic Performance for Centered Operation. ASME J Tribol 112: 692698.

16. Sawicki JT, Capaldi RJ, Adams ML (1997) Experimental and Theoretical Rotordynamic Characteristics of A Hybrid Journal Bearing. ASME J Tribol 119: 132-141

17. Yoshikawa H, Ota T, Higashino K, Nakai S (1999) Numerical Analysis on Dynamic Characteristics of Cryogenic Hydrostatic Journal Bearing. ASME J Tribol 121: 879-885.

18. Talukder HM, Stowell TB (2003) Pneumatic Hammer in An Externally Pressurized Orifice-Compensated Air Journal Bearing. Tribol Int 36: 585-591.

19. Ghosh MK, Satish MR (2003) Stability of Multilobe Hybrid Bearings with Short Sills-Part I, Tribol Int. 36: 625-632.

20. Ghosh MK, Satish MR (2003) Stability of Multilobe Hybrid Bearings with Short Sills-Part II. Tribol Int 36: 633-636.
21. Shyu SH (2006) The Parameters for THD Lubrication Flow. Chinese J Mechanics. 22: 19-26.

22. Wang N, Tsai CM, and Cha KC (2009) A Study of Parallel Efficiency of Modified Direct Algorithm Applied to Thermo-Hydrodynamic Lubrication. Chinese J Mechanics 25: 143-150.

23. Su JCT, Lie KN (2001) Rotation Effect on Hybrid Hydrostatic/Hydrodynamic Journal Bearings. Industrial Lubrication and Tribology 53: 261-269.

24. Su JCT, Lie KN (2003) Rotation Effect on Hybrid Air Journal Bearings. Tribol Int 36: 717-726.

25. Su JCT Lie KN (2006) Rotor Dynamic Instability Analysis on Hybrid Air Journal Bearings. Tribol Int 39: 238-248.

26. Otsu Y, Miyatake M, Yoshimoto S (2011) Dynamic Characteristics of Aerostatic Porous Journal Bearings with A Surface-Restricted Layer. ASME J Tribol 133 56-65. 